\title{
The Molecular Diffusion of Heat and Mass from Two Spheres
}

\author{
Abdullah Abbas Kendoush \\ Dept. of Nuclear Engineering Technology, Augusta Technical College, Augusta, Ga, USA \\ *Corresponding Author: Abdullah Abbas Kendoush, Dept. of Nuclear Engineering Technology, \\ Augusta Technical College, Augusta, Ga, USA
}

\begin{abstract}
An analytical solution to the heat conduction equation applied to two spheres was obtained. Bispherical coordinates system were used. The derived solution applies to both fluid and solid spheres. The solution revealed a decrease in the heat conduction between the two spheres when they approach one another. The solution compared well with the available theoretical models
\end{abstract}

Keywords: Heat Conduction, Two Spheres, Heat Transfer, Temperature, Analytical Solution

\section{INTRODUCTION}

Heat and mass transfer from spheres immersed in fluids occur in many engineering industries, technologies and scientific applications. Among these are: drying, adsorption, extraction, fixed and fluidized beds, cloud physics, aerosol physics, combustion of fuel droplets and cooling of spherical uranium fuel elements in certain types of nuclear reactors.

Although an individual spherical particle or bubble is rare to be found in practical systems, yet most of the information of the scientific literature deals with studies of the individual sphere or bubble. The heat

Conduction from a single sphere to the surrounding mass of fluid is given as follows (Leal 1992)

$(N u)_{s}=2$

The above equation applies also to the case of mass transfer simply by replacing Nu by the Sherwood number Sh. Morrison and Reed (1974) derived a solution for the conductive heat transfer from two touching spheres. They found that the Nusselt number of a sphere was reduced by the presence of a touching sphere according to the following

$N u=2 \ln 2$

The problem of two, three and multi-sphere or multi-bubble system are now being addressed by a number of research workers e.g. Ruzicka (2000) and Ramachandran et al. (1989) who solved the forced convective heat transfer to a linear array of three spheres by using the finite element method. In general, an increased rate of convective heat or mass transfer with sphere separation was obtained.

The molecular heat conductin and mass diffusion of the two spheres are governed by the following phenomenon: the diffusion of heat or mass from one sphere moderates the gradient of temperature and concentration around the other sphere and thus $\mathrm{Nu}$ or $\mathrm{Sh}$ are decreased with the separation distance between spheres. This phenomenon is encountered in low Reynolds number flow (e.g. Chen and Pfeiffer (1970), Aminzadeh et al. (1974) and Ramachandran and Kleinstreuer (1985).

The following conclusions were deduced from the literature review:

a. Aconfusion exists as to the increase or decrease of heat transfer with sphere spacing. (see for example Mulholand et al. (1988).

b. The majority of the theoretical research was confined to the numerical solution of the conservation equations, except for the work of Kendoush (2007).

c. Two-sphere interactions were not treated experimentally, except for the works of Wang and Liu 
(1992) of the two solid spheres, Kok (1989), and Sanada (2005) of the two bubbles.

The ideal case of the present paper serves the purpose of modeling practical situations of conductive flow of heat in a system of two spheres. The two-sphere analysis may be considered as the lowest order effect of thermal interaction of multi-spheres in a swarm. The present presentation may be considered as a complementary to that of Kendoush (2007).

\section{Theoretical Analysis}

Assume two spheres of equal radii $a$ separated by a certain distance $L$ as shown in Fig. 1. Yovanovich (1978) developed a method of dealing with heat conduction in complicated geometries. The method depends on choosing an orthogonal curvilinear coordinate system which is most appropriate for the problem at hand, and solving Laplace's equation in that coordinate system. Accordingly, the bispherical coordinate system was chosen here for the present problem. The heat conduction equation, in bispherical coordinates, is given as follows

$\frac{(\cosh \eta-\cos \theta)^{3}}{c^{2} \sin \theta}\left[\sin \theta \frac{\partial}{\partial \eta}\left(\frac{1}{\cosh \eta-\cos \theta} \frac{\partial T}{\partial \eta}\right)+\frac{\partial}{\partial \theta}\left(\frac{\sin \theta}{\cosh \eta-\cos \theta} \frac{\partial T}{\partial \theta}\right)\right]=0$

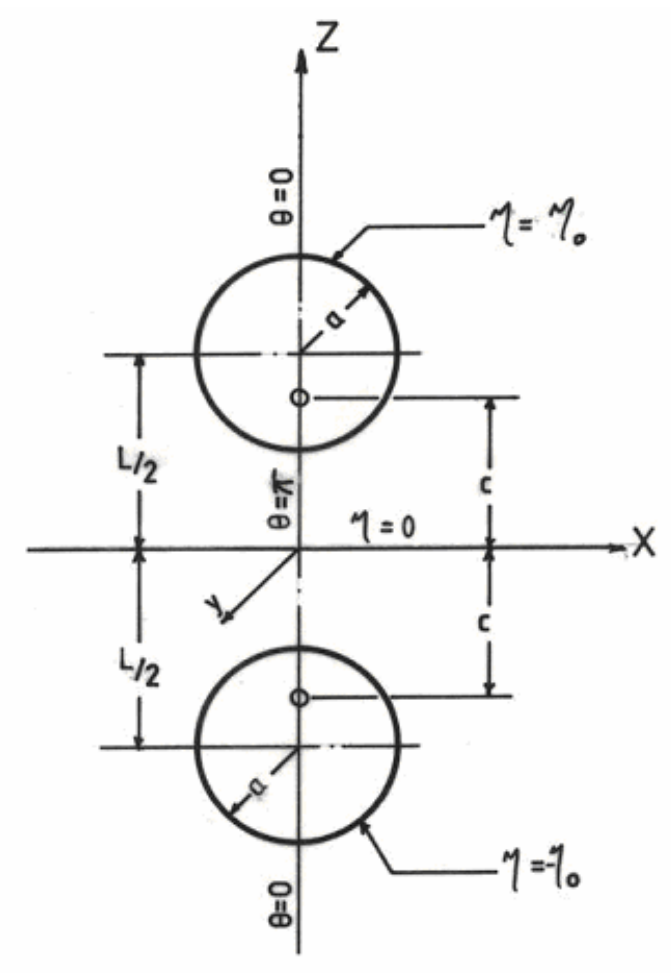

Fig1. The two spheres in bispherical coordinates.

It will be supposed that heat is generated within the two spheres at a rate such that their surface are maintained at a uniform temperature $T_{a}$. The boundary conditions, which specify the temperature and heat flow of the system become the following

$T=T_{a}$ at $\eta=\eta_{o}$

$\frac{\partial T}{\partial \eta}=0$ at $\eta=0$

$\frac{\partial T}{\partial \theta}=0$ at $\theta=0-\pi$

Laplace's Eq. (3) is solved by separation of variables. A solution of sufficient generality for our purpose is the following

$T=(\cosh \eta-\cos \theta)^{1 / 2}\left[B e^{(n+0.5) \eta}+D e^{-(n+0.5) \eta}\right] P_{n}(\cos \theta)$

where $\mathrm{B}$ and $\mathrm{D}$ are arbitrary constants and $p_{n}(\cos \theta)$ is the associated Legendre function of the first kind. Applying the boundary conditions makes the complete solution as follows 


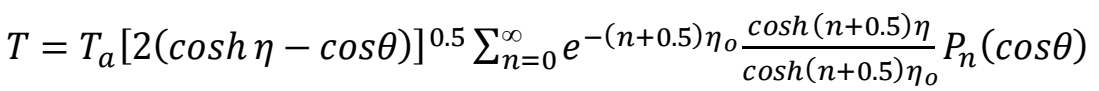

Assume that the mass of the stationary fluid round the two spheres has a constant thermal conductivity $\mathrm{k}$.

The heat flux from the spheres surfaces $S_{O}$ is given by the following

$q=\iint_{S_{o}} k g^{1 / 2}\left(\frac{\partial T}{\partial \eta}\right) n_{o} d S_{n}$

where

$$
g_{1}=c^{2} /(\cosh n-\cos \theta)^{2}
$$

and

$$
d S_{n}=\left(g_{2} g_{3}\right)^{1 / 2} d \theta=\frac{c^{2} \sin \theta}{\left(\cosh n_{0}-\cos \theta\right)^{2}} d \theta
$$

The metric coefficients of the coordinates are the following

$$
g_{1}=g_{2}
$$

And

$$
g_{3}=\frac{c^{2} \sin ^{2} \theta}{(\cosh n-\cos \theta)^{2}}
$$

The following dimensions are defined as follows: $a=c / \sinh n_{o}$ and $L=2 c / \tanh n_{o}$. The temperature gradient is obtained from Eq. (8) and substituted into Eq. (9) to get the following equation

$$
q=4 \pi a k T_{a} \sum_{n=0}^{\infty} \frac{(-1)^{n} \sinh n_{o}}{\sinh (n+1) n_{o}}
$$

Equating the above equation to $q=h\left(4 \pi a^{2}\right) T_{\alpha}$, gives the following equation

$$
N u=2 \sum_{n=0}^{\infty} \frac{(-1)^{n} \sinh n_{o}}{\sinh (n+1) n_{o}}
$$

Or the following

$$
N u=\sum_{n=0}^{\infty} \frac{(-1)^{n} \sinh \cosh ^{-1}\left(\frac{L}{2 a}\right)}{\sinh (n+1) \cosh ^{-1}\left(\frac{L}{2 a}\right)}
$$

Equation (15) reduces to the single sphere solution (that is, $\mathrm{Nu}=2$ of Eq.(1) upon increasing the value of $\eta_{\mathrm{o} o r} \mathrm{~L}$. This increase tends to make the two spheres an infinite distance apart. On the other hand, when $\eta_{0}=0.2$ (the case of almost touching spheres), we obtain from Eq. (15) the solution of Morrison and Reed [2] (that is, $\mathrm{Nu}=2 \ln 2$ of Eq. (2)). Dividing Eq. (15) by the single sphere, we get the following equation

$$
N u /(N u)_{s}=\sum_{n=0}^{\infty}(-1)^{n} \sinh \eta_{o} / \sinh (n+1) \eta_{o}
$$

\section{Dis CUSSION}

The above analyses of the two spheres apply to both fluid and solid spheres. Figure 2 shows that when the centers of the spheres are only 4 radii apart the Nusselt number is approximately 1.6 and when the centers of the spheres are 100 radii apart the Nusselt number is approximately $1 \%$ less than the limiting value of 2 . It can be deduced from Fig.2 that as the number of spheres is increased the Nusselt number becomes progressively smaller until a condition is reached in which a particular sphere is surrounded by multiple spheres of the same surface temperature. This means that a point will be reached where the Nusselt number approaches zero. Figure 2 shows a 
comparison between the present solution and that of Morrison and Reed (1974). Both solutions agree on the decrease of $\mathrm{Nu}$ number with separation but the present solution has the following merits

(i) It is in a closed form. This would enable researchers to utilize the present solution together with the earlier solution of the author (Kendoush (2007)) to obtain the complete solution of heat transfer to two bubbles vertically above one another, as follows

$$
N u=2 \sum_{n=0}^{\infty} \frac{(-1)^{n} \sinh ^{-\cosh ^{-1}\left(\frac{L}{2 a}\right)}}{\sinh (n+1) \cosh ^{-1}\left(\frac{L}{2 a}\right)}+\frac{1}{2}\left[\frac{2}{\sqrt{\pi}} \sqrt{H}\left([P e]_{A}\right)^{1 / 2}+\frac{2}{\sqrt{\pi}} \sqrt{G}\left([P e]_{B}\right)^{1 / 2}\right]
$$

where

$H=\left[1+(a / L)^{3}\right]$

and

$$
G=\left[1+\left(\frac{a}{L}\right)^{6}\right]
$$

Subscripts A and B refers to top and bottom bubbles respectively.

(ii) The present results are closer to those of Russell (1911) than Morrison and Reed (1974). Russell (1911) calculated electric charges on two spherical condensers whose surfaces were at the same potential. His results were presented in approximate formulas and tables.

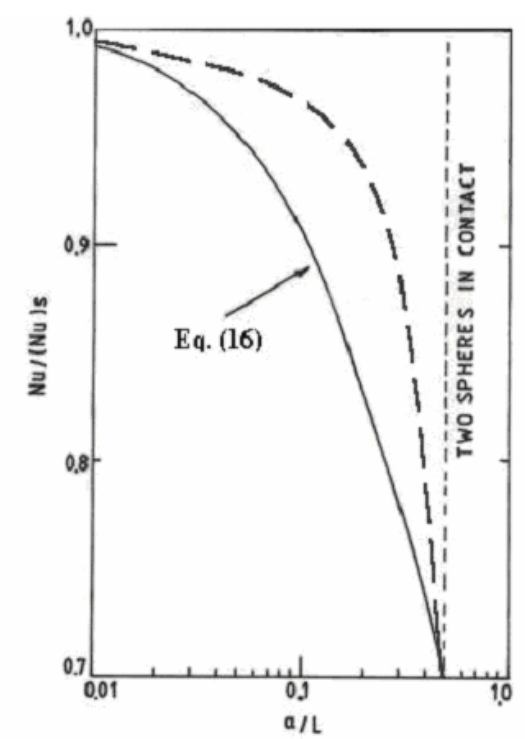

Fig2. Comparison between the present solution (-----) and that of Morrison and Reed (1974) (-----)

\section{COnClusion}

The present paper revealed and proved analytically the following phenomenon:

"A decrease in the heat conduction and mass diffusion between two solid or fluid spheres occurs when they approach one another." This is contrary to the case of heat convection Kendoush (2007).

In general, the present analytical results compared well with the available theoretical models.

\section{ACKNOWLEDGMENT}

The author wishes to dedicate this work to the living memory of the late Professor Hugh C. Simpson of the University of Strathclyde (UK), former editor and founder of the International Journal of Multiphase Flow. The author had enjoyed his brilliant lectures and had good fortune of working under his supervision. 


\section{NOMENCLATURE}

$\begin{array}{lll}\mathrm{a} & = & \text { radius of the sphere } \\ \mathrm{h} & = & \text { convective heat transfer coefficient } \\ \mathrm{k} & = & \text { thermal conductivity of the fluid } \\ \mathrm{L} & = & \text { the distance between centers of the two spheres } \\ \mathrm{Nu} & = & \text { Nusselt number }(2 a h / k) \\ \mathrm{q} & =\quad \text { the heat flux on the sphere surface } \\ \mathrm{Re} & =\quad \text { Reynolds number }(2 a \rho U / \mu) \\ T & =\quad \text { temperature } \\ T_{a}= & \text { absolute temperature of the sphere surface } \\ \eta, \theta \quad= & \text { bispherical coordinates }\end{array}$

\section{REFERENCES}

Aminzadeh, K., Al-Taha, T.R., Cornish, A.R.h., Kolansky, M.S. and Pfeiffer, R., Mass transport around two spheres at low Reynolds Number, Int. J. Heat Mass transfer, vol. 17, pp. 1425-1436, 1974.

Chen, W.C. and Pfeiffer, R., 1970, Mass transfer rates with first order homogeneous chemical reaction around two spheres, Chem. Eng. Prog. Symp. Ser., No.105, 66, pp. 109-118, 1970.

Kendoush, A.A., Enhancement of convective heat and mass transfer from two bubbles at high Reynolds number, ASME J. Heat Transf., vol.129, pp. 211-219, 2007.

Kok, J.B.W., Dynamics of gas bubbles moving through liquid, Ph.D. Thesis, University of Twent, The Netherlands, 1989.

L.G. Leal, Laminar Flow and Convective Transport Processes, Butterworth-Heinemann: Boston 1992.

Morrison, F.A. Jr. and Reed, L.D., Low Knudsen number heat transfer from two spheres in contact, ASME J. Heat Transfer, v o 1. 96, pp. 478-482, 1974.

Mulholand. J.A., Srivastava, R.K. and Wendt, J.O.L., Influence of droplet spacing on drag coefficient in no evaporating, monodisperse streams, AIAA Journal, vol. 26, pp. 1231-1245, 1988.

Rama chandran, R.S . and Kleinstreuer, C., "Mass transfer with chemical reaction in two interacting drops", Chem. Eng. Commun., vol. 36, pp. 197 -210, 1985.

Rama chandran, R.S. and Kleinstreuer, C.,Yang, T.-Y., 1989, Forced convection heat transfer of interacting spheres, Numerical Heat Transfer, vol. 15, pp. 471-487, 1989.

Russel, A., The electric stress at which ionisation begins in air, Proc. Phys. Soc., vol. 23, pp. 352-364, 1911.

Ruzicka, M.C., On bubbles rising in line, Int. J. Multiphase Flow, vol.26, pp. 1141-1181, 2000.

Sanada, T., Interaction and coalescence of bubbles in quiescent liquid, Ph.D. Thesis, Kyushu

University, Japan, 2005.

Wang, B.-X. and Liu, T., Research on hydrodynamics and heat transfer for fluid flow around heating spheres in tandem, Int. J. Heat Mass Transfer, vol. 35, pp. 307-317, 1992.

Yovanovich, M. M., Advanced Heat Conduction, Hemisphere: Washington, 1978.

Citation: Abdullah Abbas Kendoush et.al. (2018)" The Molecular Diffusion of Heat and Mass from Two Spheres", International Journal of Modern Studies in Mechanical Engineering, 4(1), pp.24-28. DOI: http://dx.doi. org/10.20431/2454-9711.0401004

Copyright: (C) 2018 Abdullah Abbas Kendoush, This is an open-access article distributed under the terms of the Creative Commons Attribution License, which permits unrestricted use, distribution, and reproduction in any medium, provided the original author and source are credited. 